\title{
Canaliculitis with isolation of Pityrosporum pachydermatis
}

\author{
A. ROMANO, ${ }^{1}$ E. SEGAL, ${ }^{2}$ AND M. BLUMENTHAL ${ }^{1}$
}

From the ${ }^{1}$ Maurice and Gabriela Goldschleger Eye Institute, Chaim Sheba Medical Centre, Tel-Hashomer, and the ${ }^{2}$ Department of Human Microbiology, Tel-Aviv University, Sackler School of Medicine, Tel-Aviv, Israel

SUMMARY A case of canaliculitis with obstruction of the lacrimal canaliculus and accumulation of conglomerates is described. Pityrosporum pachydermatis was cultured from the conglomerates. To the best of our knowledge this organism has not been previously implicated in such infections. Cure was obtained by treatment with nystatin administered topically and as an irrigation of the lacrimal pathways. The possibility that the pathogenesis of the canaliculitis and obstruction of the lacrimal pathways lies in decreased function of the lacrimal pump is discussed.

Canaliculitis is a well known clinical entity despite its relative rarity (Vaughan et al., 1965). Various organisms have been described in its aetiology, including bacteria, actinomycetes, and fungi (Donahue, 1949; Vaughan et al., 1965; Francois, 1968; Locatcher-Khorazo and Seegal, 1972; Duke-Elder, 1974). The fungi have included Aspergillus, Candida, Trichosporon, Scopulariopsis, and Cephalosporium species (Francois, 1968; Locatcher-Khorazo and Seegal, 1972). The present work describes a case of a complete occlusion of the lacrimal canaliculus due to a deposit of concrement from which Pityrosporum pachydermatis was cultured. To the best of our knowledge this species of fungus has not been previously implicated in such infections.

\section{Case report}

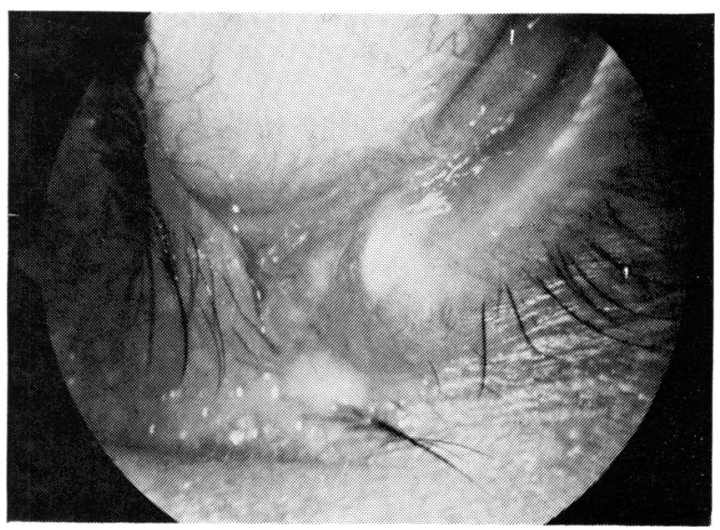

Fig. 1 Button of yellow purulent secretion on the lacrimal punctum

punctum (Fig. 1). There was a symblepharon of the conjunctiva connecting the margin of the lacrimal punctum with the plica, with traction on the punctum. Throughout the examination a continuous epiphora was observed.

The lacrimal pathways of both eyes were rinsed with saline, which passed freely to the pharynx, raising the suspicion of a canalicular infection. Conglomerates of yellow material (Fig. 2) were obtained by pressure with the finger on the area of the lacrimal duct in the direction of the canaliculus and lacrimal punctum. This material was cultured for bacterial and fungal agents. These cultures yielded Gram-positive micrococci and a yeast-like
Address for reprints: Dr A. Romano, Department of Ophthalmology, Tel Aviv University, Sackler School of Medicine, Chaim Sheba Medical Centre, Tel-Hashomer, Israel 


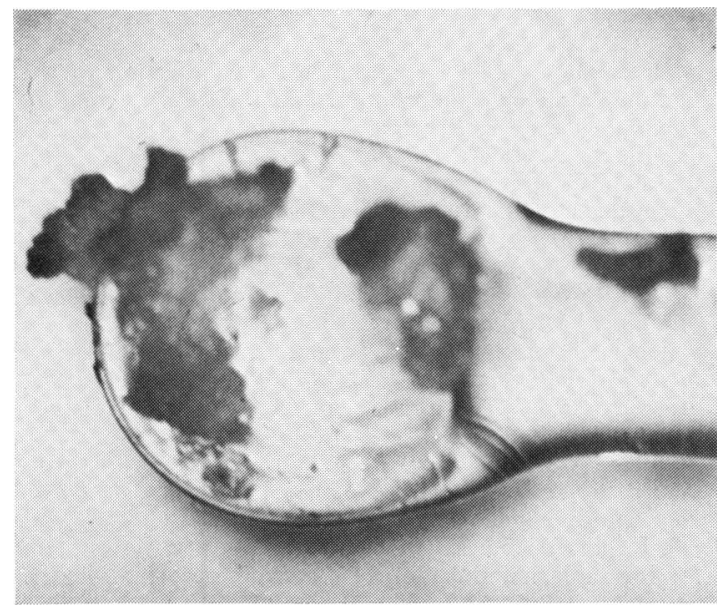

Fig. 2 Conglomerates of yellow material pressed out from the canaliculus

organism growing on Sabouraud's dextrose agar (Figs. 3 and 4) both at room temperature and at $37^{\circ} \mathrm{C}$. The microscopic examination of subcultures from cornmeal agar revealed the presence of blastospores with mycelium.

Tests of assimilation of glucose, maltose, sucrose, lactose, galactose, xylose, trehalose, raffinose inositol melibiose, and cellobiose using the classical Wickerham method (Lodder, 1971) were negative. A weak assimilation of glucose was seen by an auxinographic technique (Huppert et al., 1975; Segal and Ajello, 1976). Fermentation of glucose, maltose, sucrose, and lactose and the urease test gave negative results. Applying an auxinographic method for nitrogen compounds assimilation revealed the assimilation of ammonium sulphate and not of potassium nitrate. These various morphological and biochemical characteristics were confirmed by the Fungus Diagnostic Branch of the Mycology Division at the Center for Disease Control in Atlanta, Georgia, USA, where the organism was identified as Pityrosporum pachydermatis.

Treatment was initiated with the antifungal agent nystatin, administered as drops (7000 units nystatin/ $\mathrm{ml}$ saline), 2 drops 6 times a day for a month, and as an irrigation of the lacrimal pathways twice a week. In addition, the symblepharon was released. After a month of this treatment a cure was obtained.

\section{Discussion}

Accumulation of material in the form of conglomerates is found in canaliculitis due to actinomyces (Francois, 1968). Wolter and Deitz (1963) described

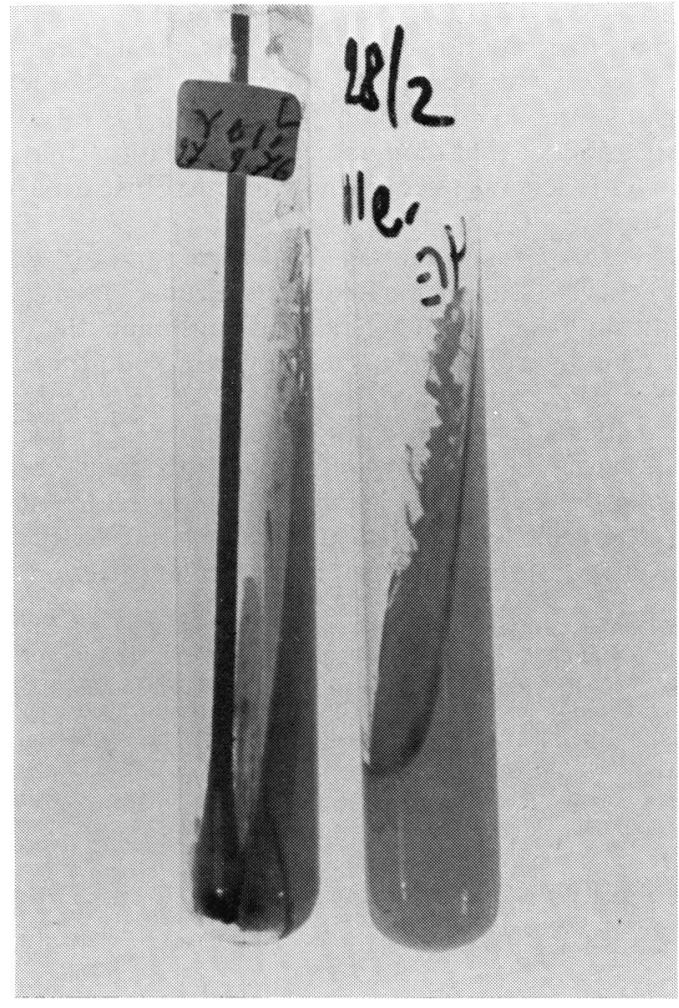

Fig. 3 Yeast-like culture isolated from conglomerates

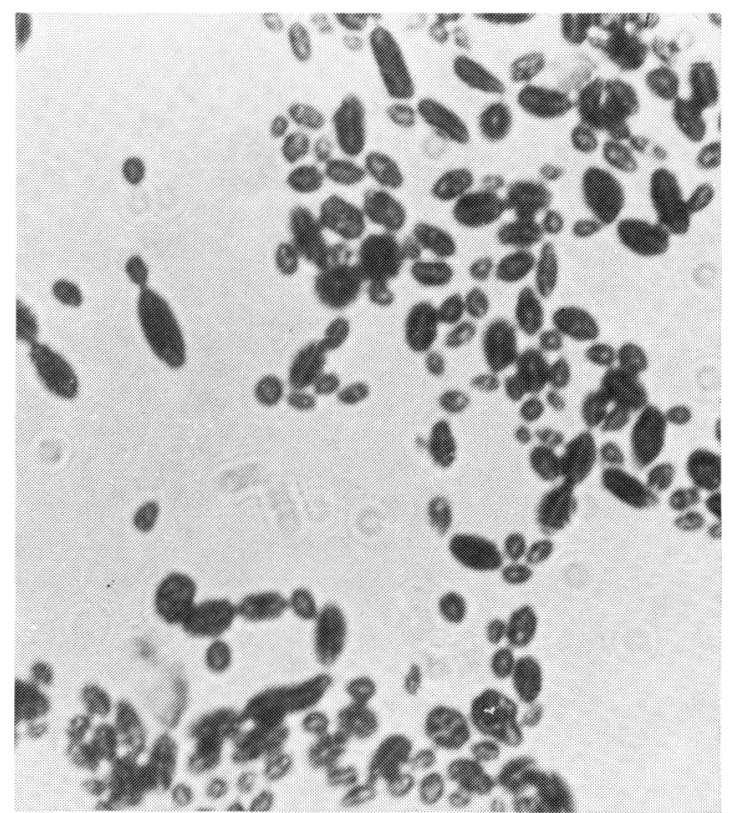

Fig. 4 Budding yeast cells from Sabouraud's dextrose agar culture 
a case of dacryocystitis due to Candida albicans with concrement formation in the lacrimal sac. The isolation of Pityrosporum pachydermatis from the conglomerates in the canaliculus of our case raises the possibility that conglomerate formation may be a more general phenomenon in microbial canaliculitis.

Pityrosporum species ( $P$. orbiculare and/or $\boldsymbol{P}$. ovale) have been associated with ocular disease, specifically seborrhoeic blepharitis, although there is no definite proof of their aetiological role in these diseases (Parunovic and Halde, 1967; Francois, 1968; Locatcher-Khorazo and Seegal, 1972). The isolation of $P$. pachydermatis from the conglomerates in our case, and the cure following treatment with a specific antifungal substance, point to the involvement of this organism in the ocular infection.

We wish to express our appreciation to the staff of the Fungus Diagnostic Branch, Mycology Division, Center for Disease Control, Atlanta, and Professor D. Ahearn, Department of Biology, Georgia State University, Atlanta, Georgia, USA, for their assistance in identification of the organism and valuable information.

\section{References}

Donahue, H. C. (1949). Unusual mycotic infection of the lacrimal canaliculi and conjunctiva. American Journal of Ophthalmology, 32, 207-210.

Duke-Elder, S. (1974). System of Ophthalmology, vol. 13, pp. 693-698. Henry Kimpton: London.

Francois, J. (1968). Les Mycoses Oculaires, pp. 42-49, 139 , Masson: Paris.

Huppert, M., Harper, G., Sung, S. H., and De Lanerolle, V. (1975). Rapid methods for identification of yeasts. Journal of Clinical Microbiology, 2, 21-34.

Locatcher-Khorazo, D., and Seegal, B. C. (1972). Microbiology of the Eye, pp. 72-73, 216-226. Mosby: St. Louis.

Lodder, J. (1971). The Yeasts, pp. 65-84. North Holland: Amsterdam.

Parunovic, A., and Halde, C. (1967). Pityrosporum orbiculare: its possible role in seborrheic blepharitis. American Journal of Ophthalmology, 63, 815-820.

Segal, E., and Ajello, L. (1976). Evaluation of a new system for the rapid identification of clinically important yeasts. Journal of Clinical Microbiology, 4, 157-159.

Vaughan, D., Cook, R., and Asbury, T. (1965). General Ophthalmology, pp. 62-64. Lange Medical Publications: Los Altos.

Wolter, J. R., and Deitz, M. R. (1963). Candidiasis of the lacrimal sac. American Journal of Ophthalmology, 55, 153-155. 